\title{
Clinical Characteristics of Advanced Non-Small Cell Lung Cancer with EGFR Exon 20 Insertions
}

\author{
Chie Morita \\ National Cancer Center Hospital \\ Tatsuya Yoshida ( $\square$ tatyoshi@ncc.go.jp) \\ National Cancer Center Hospital https://orcid.org/0000-0003-4896-5824 \\ Masayuki Shirasawa \\ National Cancer Center Hospital \\ Ken Masuda \\ National Cancer Center Hospital \\ Yuji Matsumoto \\ National Cancer Center Hospital \\ Yuki Shinno \\ National Cancer Center Hospital \\ Shigehiro Yagishita \\ National Cancer Research institute \\ Yusuke Okuma \\ National Cancer Center Hospital \\ Yasushi Goto \\ National Cancer Center Hospital \\ Hidehito Horinouchi \\ National Cancer Center Hospital \\ Noboru Yamamoto \\ National Cancer Center Hospital \\ Noriko Motoi \\ National Cancer Center Hospital \\ Yasushi Yatabe \\ National Cancer Center Hospital \\ Yuichiro Ohe \\ National Cancer Center Hospital
}

\section{Research Article}

Keywords: Non-small cell lung cancer, Epidermal growth factor receptor mutation, Epidermal growth factor receptor exon 20 insertions

Posted Date: June 22nd, 2021

DOI: https://doi.org/10.21203/rs.3.rs-609572/v1

License: 두 (i) This work is licensed under a Creative Commons Attribution 4.0 International License. Read Full License 


\begin{abstract}
Background: Epidermal growth factor receptor (EGFR) exon 20 insertion mutations account for $4 \%-10 \%$ of all EGFR mutations in patients with non-small cell lung cancer (NSCLC). However, data on the differences in clinical characteristics between patients with EGFR exon 20 insertion mutations and major mutations (exon 19 deletion and L858R) are limited.

Methods: We retrospectively reviewed advanced NSCLC patients with EGFR mutations, including EGFR exon 20 insertions and major mutations, who were treated with systemic therapy between January 2011 and December 2019.

Results: We identified 23 patients with EGFR exon 20 insertions and 534 patients with EGFR mutations. Among patients with exon 20 insertion, the median age was 60 years (range: $27-88$ years), and females and never smokers were predominant. The clinical characteristics of patients with exon 20 insertions were similar to those with major EGFR mutations. Regarding the clinical outcomes in patients with exon 20 insertions, 17 patients received platinum doublet as first-line therapy, and the overall response rate (ORR) and median progression-free survival (mPFS) were $11.8 \%$ and $8.9 \%$. Additionally, eight patients received anti-PD-1 antibodies and seven patients received EGFR-tyrosine kinase inhibitors (TKIs) in any-line therapy, and their ORR and mPFS were $0 \%, 25 \%$ and $2.2,3.1$ months, respectively. Overall survival was significantly shorter in patients with exon 20 insertions than in those with EGFR major mutations (29.3 vs. 43.4 months, $p=0.04)$.
\end{abstract}

Conclusions: The clinical outcomes in patients with exon 20 insertions were not satisfactory compared to those in patients with major EGFR mutations.

\title{
Introduction
}

Lung cancer is the leading cause of cancer-related death worldwide, with non-small lung cancer (NSCLC) accounting for approximately $80 \%$ of all lung cancer deaths [1]. The frequency of epidermal growth factor receptor (EGFR) mutations has been reported to be $47.9 \%$ in adenocarcinoma and $4.6 \%$ in squamous cell carcinoma among Asian populations and $19.2 \%$ in adenocarcinoma and $3.3 \%$ in squamous cell carcinoma among Western populations [2]. EGFR mutations mainly occur between exons 18 and 21 , and the most common genetic mutation is the deletion of exon 19 and L858R in exon 21, which accounts for 85-95\% of all EGFR mutations [3]. EGFR mutations are commonly found in never smokers, women, and patients with adenocarcinoma $[4,5]$. Most patients with these EGFR mutations respond to treatment with EGFR tyrosine kinase inhibitors (TKIs) such as gefitinib, erlotinib, afatinib, and osimertinib, with median progression-free survivals (mPFS) of 9.2-18.9 months[6-10]. [11]

Exon 20 insertion mutations account for about $4-10 \%$ of all EGFR mutations, and is associated with a lack of sensitivity to the aforementioned EGFR-TKIs [3, 12-14]. The standard treatment for patients with exon 20 insertion is systemic chemotherapy, which is similar to the treatment of other NSCLC cases without driver mutations $[15,16]$. However, novel targeted therapies against NSCLC with exon 20 insertion mutations, such as poziotinib [17], mobocertinib (TAK-788) [18], and amivantamab (JNJ-61186372) [19] have been developed.

Few studies have focused on the differences in clinical characteristics between patients with exon 20 insertions and those with EGFR major mutations. Our study therefore aimed to clarify the clinical characteristics and outcomes, including the efficacy of systemic treatment in patients with exon 20 insertion mutations, compared with those with major EGFR mutations.

\section{Patient And Methods Subjects}

We retrospectively reviewed the records of advanced NSCLC patients with exon 20 insertion mutations treated with systemic chemotherapy, and those with EGFR major mutations (e.g., deletion in exon 19 and L858R in exon 21) treated with EGFR-TKIs as initial treatment at the National Cancer Center Hospital in Japan between January 2011 and December 2019. We collected data on patient characteristics, variants of exon 20 insertion, and clinical outcomes.

\section{Detection of EGFR mutation including exon 20 insertion mutations}

The diagnosis of EGFR mutation including exon 20 insertion was performed based on PCR-based methods (Scorpion-Arms methods and Cobas ${ }^{\circledR}$ EGFR Mutation Test v2; Roche Diagnostics, Basel, Switzerland) and next-generation sequencing (NGS), such as

Page 2/11 


\section{Statistical Analysis}

To evaluate the differences in clinical characteristics between the patients, Fisher's exact test was performed. The treatment effect was evaluated based on the Response Evaluation Criteria in Solid Tumors (RECIST version 1.1) [20]. The overall response rate (ORR) was defined as the percentage of patients with the best overall response of complete response (CR) or partial response (PR). We also used the Kaplan-Meier method to investigate PFS and overall survival (OS). OS was defined as the time from the date of diagnosis to death. PFS was defined as the time from the start of treatment to disease progression or death and was censored on the date the patient was last known as progression-free. All statistical analyses were performed using the EZR ver. 1.41 [21]. This study was approved by the Ethics Committee of the National Cancer Center Hospital (2015 - 355).

\section{Results}

\section{Patient Characteristics}

We identified 23 patients with EGFR exon 20 insertions and 534 patients with EGFR major mutations, including 285 patients with an exon 19 deletion and 249 patients with an L858R mutation in exon 21. Patient characteristics according to EGFR mutation status are shown in Table 1. Patients with EGFR exon 20 insertions were significantly younger than those with major EGFR mutations (median age: 60 vs. 66 years). There were no significant differences in baseline characteristics between patients with exon 20 insertions and major EGFR mutations, except for age. Regarding the metastatic spread, bone (21.6\%) was the most common metastatic site in patients with EGFR exon 20 insertions, followed by the central nervous system (CNS) (13.0\%), liver (17.4\%). Patients with intrathoracic metastases were more common in patients with EGFR exon 20 mutations (52.2\%) than in those with EGFR major mutations (35.2\%), although the differences were not significant. Of the 23 patients with an EGFR exon 20 insertion, four were assessed for variants of EGFR exon 20 insertion (Table 2). 
Table 1

Characteristics of patients harboring EGFR exon 20 insertions and major mutations.

\begin{tabular}{|c|c|c|c|c|c|}
\hline & \multirow{2}{*}{$\begin{array}{l}\text { Exon } 20 \text { insertions } \\
N=23\end{array}$} & \multicolumn{3}{|c|}{ EGFR major mutations } & \multirow[t]{2}{*}{ P-value } \\
\hline & & All $(N=534)$ & Ex19 del $(\mathrm{N}=285)$ & L858R $(\mathrm{N}=249)$ & \\
\hline Age $(y)$, mean (SD) & $60(27-88)$ & $66(28-88)$ & $65(32-88)$ & $68(28-87)$ & 0.017 \\
\hline$\geq 75, \mathrm{n}(\%)$ & $3(13.0)$ & $125(23.4)$ & $57(20.0)$ & $68(27.3)$ & 0.318 \\
\hline$<75, \mathrm{n}(\%)$ & $20(87.0)$ & 409 (76.6) & $228(80.0)$ & $181(72.7)$ & \\
\hline \multicolumn{6}{|l|}{ Sex, n (\%) } \\
\hline Female & $18(78.3)$ & $338(63.3)$ & $173(60.7)$ & $165(66.3)$ & 0.233 \\
\hline Male & $5(21.7)$ & $196(36.7)$ & $112(39.3)$ & $84(33.7)$ & \\
\hline \multicolumn{6}{|l|}{ Histology, n (\%) } \\
\hline Ad & $22(95.7)$ & $521(97.6)$ & $277(97.2)$ & $244(98.0)$ & 0.450 \\
\hline Others & $1(4.3)$ & $13(2.4)$ & $8(2.8)$ & $5(2.0)$ & \\
\hline \multicolumn{6}{|l|}{ Smoking, n (\%) } \\
\hline Never & $15(65.2)$ & $313(58.6)$ & $152(53.3)$ & $161(64.6)$ & 0.847 \\
\hline Current/Former & $8(34.8)$ & $218(40.8)$ & $132(46.3)$ & $86(34.5)$ & \\
\hline Unknown & 0 & $3(0.6)$ & $1(0.4)$ & $2(0.8)$ & \\
\hline Stage, n (\%) & $16(69.6)$ & 309 (57.9) & $173(60.7)$ & $136(54.6)$ & 0.290 \\
\hline IVA/IVB & $7(30.4)$ & $225(42.1)$ & $112(39.3)$ & $113(45.4)$ & \\
\hline \multicolumn{6}{|l|}{ recurrence } \\
\hline \multicolumn{6}{|l|}{ Metastasis, n (\%) } \\
\hline Bone & $5(21.6)$ & $225(42.1)$ & $128(44.9)$ & $97(39.0)$ & 0.055 \\
\hline CNS & $3(13.0)$ & $134(25.1)$ & $76(26.7)$ & $58(23.3)$ & 0.225 \\
\hline Liver & $4(17.4)$ & $61(11.4)$ & $37(13.0)$ & $24(9.6)$ & 0.330 \\
\hline Intrathoracic disease & $12(52.2)$ & $188(35.2)$ & $94(33.0)$ & $94(37.8)$ & 0.322 \\
\hline
\end{tabular}


Table 2

Response of systemic therapy in patients with EGFR exon 20 insertions and major mutations.

\begin{tabular}{|lllllllll|}
\hline Types of Systemic therapy & & PR & SD & PD & NE & ORR & $p$-value \\
\hline Platinum doublet chemotherapy & Ex 20ins* $(\mathrm{N}=17)$ & 2 & 13 & 1 & 1 & $11.8 \%(1.5-36.4 \%)$ & 0.75 \\
& Major $(\mathrm{N}=163)$ & 35 & 82 & 41 & 5 & $21.5 \%(15.4-28.6 \%)$ & \\
\hline EGFR-TKIs & Ex 20ins $(\mathrm{N}=7)$ & 0 & 1 & 5 & 1 & $0 \%(0-3.5 \%)$ & 0.003 \\
& Major* $(\mathrm{N}=534)$ & 309 & 148 & 34 & 43 & $57.9 \%(53.5-62.1 \%)$ & \\
\hline Anti-PD-1 Antibody & Ex 20ins $(\mathrm{N}=8)$ & 2 & 2 & 4 & 0 & $25 \%(3.2-65.1 \%)$ & 0.61 \\
& Major $(\mathrm{N}=38)$ & 6 & 9 & 23 & 0 & $15.8 \%(6.0-31.3 \%)$ & \\
\hline $\begin{array}{l}\text { PR, partial response; SD, stable disease; PD, progressive disease; NE, non-evaluable; EGFR-TKI, epidermal growth factor receptor- } \\
\text { tyrosine kinase inhibitor; Ex 20 ins, exon20 insertions; PD-1, programmed cell death-1; * first-line setting. }\end{array}$ & \\
\hline
\end{tabular}

\section{Clinical outcomes in patients with exon 20 insertions}

Of the 23 patients, 17 received platinum doublet chemotherapy, including two patients who received platinum doublet chemotherapy in combination with anti-PD-1 antibody, and 1 in combination with EGFR-TKIs. Other first-line treatments were as follows: four pembrolizumab, one EGFR-TKI, and one pemetrexed monotherapy (Table 3). The ORR and mPFS of first-line platinum doublet chemotherapy in patients with EGFR exon 20 insertions were $11.8 \%(95 \% \mathrm{Cl}, 1.5$ to 36.4$)$, and 8.9 months (95\% $\mathrm{Cl}, 5.0$ to 17.3$)$, compared with ORR of $21.5 \%(95 \% \mathrm{Cl}, 15.4$ to 28.6$)$ and PFS of 5.5 months ( $95 \% \mathrm{Cl}, 4.6$ to 6.2$)$ in patients with EGFR major mutations (ORR: $p=0.75$; PFS: $p=0.01$, Table 2 and Fig. $1 \mathrm{~A}$ ).

Table 3

First-line treatment regimens in patients with exon20 insertion mutation.

\begin{tabular}{|lc|}
\hline & $\mathbf{N}(\%)$ \\
\hline Platinum doublet( $\mathrm{n}=17)$ & \\
\hline CDDP(CBDCA) + PEM & $9(39.1)$ \\
\hline CBDCA + nab-PTX & $1(4.3)$ \\
\hline CDDP(CBDCA $)+$ PEM + BEV & $4(17.4)$ \\
\hline CBDCA + PTX + BEV + Atezolizumab & $1(4.3)$ \\
\hline CBDCA + PEM + Pembrolizumab & $1(4.3)$ \\
\hline CBDCA + PEM + Gefitinib & $1(4.3)$ \\
\hline EGFR-TKIs $(n=1)$ & $1(4.3)$ \\
\hline Erlotinib & \\
\hline Anti-PD-1 monotherapy( $=4)$ & $4(17.4)$ \\
\hline Pembrolizumab & \\
\hline Other( $(n=1)$ & $1(4.3)$ \\
\hline PEM & \\
\hline CDDP, Cisplatin; CBDCA, Carboplatin; PEM, Pemetrexed; nab-PTX, nab-Paclitaxel; BEV, Bevacizumab. \\
\hline
\end{tabular}

Over the clinical course in patients with EGFR exon 20 insertions, 7 and 8 patients received EGFR-TKIs and anti-PD-1 antibody monotherapy, respectively. The differences in the ORR and PFS between exon 20 and major mutation patients in the EGFR-TKI and anti-PD-1 antibody monotherapy are shown in Table 2 and Fig. 1B-C. The ORR and mPFS of EGFR-TKIs were $0 \%, 2.2$ months $(95 \% \mathrm{Cl}$, 1.1 to NA) in patients with EGFR exon 20 insertions and $57.9 \%(95 \% \mathrm{Cl}, 53.5$ to 62.1$), 13.6$ months $(95 \% \mathrm{Cl}, 12.6$ to 14.9$)$ in those with EGFR major mutation (ORR: $p=0.003$ and, PFS: $p=0.08)$. ORR and PFS of anti-PD-1 antibody monotherapy was $25 \%(95 \% \mathrm{Cl}, 3.2$ to 
65.1), 3.1 months ( $95 \% \mathrm{Cl}, 0.7$ to 6.0 ) in patients with EGFR exon 20 insertions and $15.8 \%(95 \% \mathrm{Cl}, 6.0$ to 31.3$), 2.2$ months $(95 \% \mathrm{Cl}, 1.5$ to 3.4) in those with EGFR major mutation (ORR: $\mathrm{P}=0.61$ and, PFS: $p=0.80$ ). The median overall survival in patients with EGFR exon 20 insertions was 29.3 months ( $95 \%$ confidence interval [Cl]: 14.1). On the other hand, OS in patients with EGFR major mutations who received EGFR-TKls was 43.4 months ( $95 \% \mathrm{Cl}$ : 38.7 to 54.2). Patients with exon 20 insertions had a significantly shorter OS than those with EGFR mutations ( $p=0.04$, Figure. 2). The clinical outcomes of the four patients with the identified variants are shown in

\section{Supplemental Table 1.}

\section{Discussion}

We found that there were no significant differences in clinical characteristics, including the distribution of metastatic sites between patients with EGFR exon 20 insertion and major mutations. Additionally, we showed that the ORR and PFS of patients treated with platinum-based chemotherapy were $11.8 \%$ and $8.9 \%$, respectively. The OS of patients with EGFR exon 20 insertions was significantly shorter than in patients with major mutations who received EGFR-TKIs as initial treatment.

Very few reports have focused on the differences in clinical characteristics between major and minor EGFR mutations. Previous studies have shown that EGFR exon 20 insertion is more likely to occur in never or light smoking patients and those with adenocarcinomas [22, 23]. In our study, however, there were no differences in age, sex, smoking history, histology, or stage at diagnosis between the two groups.

EGFR exon 20 insertions are related to the intrinsic resistance to EGFR-TKIs compared with EGFR major mutations, such as exon 19 deletion and L858R in exon $21[3,12,24]$. Due to the limited efficacy of EGFR-TKIs, platinum combination chemotherapy is still the standard therapy for patients with exon 20 insertion. Previous studies have reported that mPFS was 4.2 to 6.4 months and OS was 16.4 to 29.4 months, which were similar to our data [16] [25, 26]. Additionally, OS in patients with exon 20 insertion was significantly shorter than that in patients with major mutations.

However, the clinical efficacy of EGFR-TKIs in patients with EGFR exon 20 insertion has been reported to differ according to the variant [22]. EGFR p.A763_Y764insF-QEA is considered to be more sensitive to first-to third-generation EGFR-TKIs than other variants in exon 20 insertion mutations [22, 27, 28]. In our study, only four patients had detailed information on insertion variants, and variants that are reportedly sensitive to EGFR-TKIs were not included. Although, in the current clinical procedure, we did not necessarily obtain detailed variant information, and the frequency of sensitive variants seems quite low, our results strongly support that conventional EGFR-TKIs are of little use for EGFR exon 20 insertion.

We also evaluated the efficacy of anti-PD-1/PD-L1 agents in NSCLC with EGFR exon 20 mutations. In general, anti-PD-1/PD-L1 antibodies are poorly effective in EGFR-mutated NSCLC compared with those without EGFR mutations [29-31]. However, recent studies have reported that patients with exon 20 insertions showed better clinical outcomes with anti-PD-1 antibody compared with those with EGFR major mutations [32]. In this study, the ORR and mPFS of the anti-PD-1 antibody were $25 \%$ and 3.1 months (95\% Cl: 0.7-6.0. The therapeutic effect is not sufficient, and more specific treatment for exon20 insertion is desirable.

Recently, novel targeted therapies against exon 20 insertion mutations, such as poziotinib, mobocertinib, and amivantmab have been developed [17-19]. Poziotinib, a potent TKI against EGFR and HER2 exon 20 insertion mutations, showed an ORR of 15-44\% and PFS of 4.2-5.5 months in the phase II trial $[33,34]$. Mobocertinib is an EGFR-TKI with potent and selective preclinical inhibitory activity against EGFR exon 20 insertions, with an ORR of $43 \%$ and PFS of 7.3 months in a phase II trial. A phase III trial comparing mobocertinib with platinum-based chemotherapy as first-line therapy is currently ongoing (NCT04129502) [35]. Amivantamab is an anti-EGFR-MET bispecific antibody that can target diseases driven by both EGFR and MET, and has shown therapeutic efficacy in patients with a variety of mutations, including C797S, T790M, EGFR exon20 insertion, and MET amplification. Amivantamab showed a response rate of $36 \%$ and a PFS of 8.3 months in a Phase II/III study [19]. A study is planned for patients with advanced exon 20 insertion mutations, with carboplatin and pemetrexed with and without amivantamab (NCT04538664).

This study has some limitations. First, it is a single-center, retrospective study with a small sample size as patients with EGFR exon 20 mutations are rare. Additionally, genetic variants of exon 20 insertion were assessable in only four patients, as PCR-based testing showed only the presence of exon 20 insertion, not variant types.

In conclusion, the effectiveness of EGFR-TKIs and anti-PD-1 antibodies is not sufficient. The development of an EGFR exon 20 specific treatment is therefore warranted to improve patient survival.

Page 6/11 


\section{Declarations}

\section{Funding}

No funds, grants, or other support was received.

\section{Conflict of interest.}

Dr. Yoshida has received grants and personal fees from AstraZeneca, Bristol-Myers Squibb, grants from Abbvie, MSD, Ono Pharmaceutical, Takeda Pharmaceutical, and personal fees from Chugai, Novartis. Dr. Matsumoto has received grants from Grant-inAid for Scientific Research on Innovative Areas, Hitachi High-Technologies, Hitachi, Ltd., National Cancer Center Research and Development Fund, and personal fees from AMCO INC., AstraZeneca, COOK, Olympus. Dr. Okuma has received grants from Abbvie. Dr. Goto has received grants and personal fees from Bristol-Myers Squibb, Daiichi- Sankyo, Eli Lilly, Guardant Health, MSD, Novartis, Ono Pharmaceutical, Pfizer, Taiho Pharmaceutical, grants from Kyorin, and personal fees from AstraZeneca, Boehringer Ingelheim, Chugai, Illumina. Dr. Horinouchi has received grants and personal fees from AstraZeneca, BMS, Chugai, Eli Lilly, MSD, Taiho Pharmaceutical, Ono Pharmaceutical, and grants from Astellas, Genomic Health, Merck Serono. Dr. Yamamoto has received grants and personal fees from BMS, Boehringer Ingelheim, Chugai, Eisai, Eli Lilly, Ono Pharmaceutical, Pfizer, Takeda Pharmaceutical, grants from Astellas, Bayer, Chiome Bioscience Inc., Daiichi-Sankyo, GSK, Janssen Pharma, Kyowa-Hakko kirin, MSD, Merck, Novartis, Otsuka, Taiho Pharmaceutical, Quintiles, Sumitomo Dainippon, and personal fees from AstraZeneca, Otsuka, Cimic, Sysmex. Dr. Motoi has received grants and personal fees from Ono Pharmaceutical, Roche Diagnostics, grants from NEC, personal fees from AstraZeneca, Beckton Dickinson Japan, Covidien Japan Inc, Miraca Life Sciences, MSD, Novartis, Taiho Pharmaceutical. Dr. Yatabe has received personal fees from Archer, AstraZeneca, Chugai, Dako-Agilent, MSD, Novartis, Pfizer, Thermo-Fisher Science, Ventana-Roche. Dr. Ohe has received grants and personal fees from AstraZeneca, Bristol-Myers Squibb, Chugai, Eli Lilly, Janssen Pharma, Kyorin, MSD, Nippon Kayaku, Novartis, Ono Pharmaceutical, Pfizer, Taiho Pharmaceutical, Takeda Pharmaceutical, grants from Kissei, personal fees from Boehringer Ingelheim, Celtrion. The remaining authors declare no competing interests.

\section{Availability of data and material}

The datasets generated during and/or analysed during the current study are available from the corresponding author on reasonable request.

\section{Code availability}

Not applicable.

\section{Authors' contributions}

All authors contributed to the study conception and design. Material preparation, data collection and analysis were performed by Chie Morita, Masayuki Shirasawa and Tatsuya Yoshida. The first draft of the manuscript was written by Chie Morita and all authors commented on previous versions of the manuscript. All authors read and approved the final manuscript.

\section{Ethics approval}

This study was performed in line with the principles of the Declaration of Helsinki. Approval was granted by the Ethics Committee of National Cancer Center Hospital in Japan (2015-355).

\section{Consent to participate}

Informed consent was obtained from all individual participants included in the study.

\section{Consent for publication}

Patients has consented regarding publishing their data.

\section{References}

1. Hoffman PC, Mauer AM, Vokes EE. Lung cancer. Lancet. 2000;355(9202):479-85. 
2. Dearden $\mathrm{S}$, et al. Mutation incidence and coincidence in non small-cell lung cancer: meta-analyses by ethnicity and histology (mutMap). Ann Oncol. 2013;24(9):2371-6.

3. Yasuda H, Kobayashi S, Costa DB. EGFR exon 20 insertion mutations in non-small-cell lung cancer: preclinical data and clinical implications. Lancet Oncol. 2012;13(1):e23-31.

4. Lan Q, et al. Genome-wide association analysis identifies new lung cancer susceptibility loci in never-smoking women in Asia. Nat Genet. 2012;44(12):1330-5.

5. Marchetti A, et al. EGFR mutations in non-small-cell lung cancer: analysis of a large series of cases and development of a rapid and sensitive method for diagnostic screening with potential implications on pharmacologic treatment. J Clin Oncol. 2005;23(4):857-65.

6. Zhou C, et al. Erlotinib versus chemotherapy as first-line treatment for patients with advanced EGFR mutation-positive non-smallcell lung cancer (OPTIMAL, CTONG-0802): a multicentre, open-label, randomised, phase 3 study. Lancet Oncol. 2011;12(8):73542.

7. Rosell R, et al. Erlotinib versus standard chemotherapy as first-line treatment for European patients with advanced EGFR mutationpositive non-small-cell lung cancer (EURTAC): a multicentre, open-label, randomised phase 3 trial. Lancet Oncol. 2012;13(3):23946.

8. Soria JC, et al. Osimertinib in Untreated EGFR-Mutated Advanced Non-Small-Cell Lung Cancer. N Engl J Med. 2018;378(2):113-25.

9. Sequist LV, et al. Phase III study of afatinib or cisplatin plus pemetrexed in patients with metastatic lung adenocarcinoma with EGFR mutations. J Clin Oncol. 2013;31(27):3327-34.

10. Mitsudomi T, et al. Gefitinib versus cisplatin plus docetaxel in patients with non-small-cell lung cancer harbouring mutations of the epidermal growth factor receptor (WJTOG3405): an open label, randomised phase 3 trial. Lancet Oncol. 2010;11(2):121-8.

11. Maemondo M, et al. Gefitinib or chemotherapy for non-small-cell lung cancer with mutated EGFR. N Engl J Med. 2010;362(25):2380-8.

12. Vyse S, Huang PH. Targeting EGFR exon 20 insertion mutations in non-small cell lung cancer. Signal Transduct Target Ther. 2019;4:5.

13. van Veggel $B$, et al. Osimertinib treatment for patients with EGFR exon 20 mutation positive non-small cell lung cancer. Lung Cancer. 2020;141:9-13.

14. Yang JC, et al. Clinical activity of afatinib in patients with advanced non-small-cell lung cancer harbouring uncommon EGFR mutations: a combined post-hoc analysis of LUX-Lung 2, LUX-Lung 3, and LUX-Lung 6. Lancet Oncol. 2015;16(7):830-8.

15. Wu JY, Yu CJ, Shih JY. Effectiveness of Treatments for Advanced Non-Small-Cell Lung Cancer With Exon 20 Insertion Epidermal Growth Factor Receptor Mutations. Clin Lung Cancer. 2019;20(6):e620-30.

16. Byeon S, et al. Clinical Outcomes of EGFR Exon 20 Insertion Mutations in Advanced Non-small Cell Lung Cancer in Korea. Cancer Res Treat. 2019;51(2):623-31.

17. Le X, et al. Poziotinib shows activity and durability of responses in subgroups of previously treated EGFR exon 20 NSCLC patients. J Clin Oncol. 2020;38(15_suppl):9514-4.

18. Horn L, et al. Indirect comparison of TAK-788 vs real-world data outcomes in refractory non-small cell lung cancer (NSCLC) with EGFR exon 20 insertions. J Clin Oncol. 2020;38(15_suppl):9580-0.

19. Park K, et al. Amivantamab (JNJ-61186372), an anti-EGFR-MET bispecific antibody, in patients with EGFR exon 20 insertion (exon20ins)-mutated non-small cell lung cancer (NSCLC). J Clin Oncol. 2020;38(15_suppl):9512-2.

20. Eisenhauer EA, et al. New response evaluation criteria in solid tumours: revised RECIST guideline (version 1.1). Eur J Cancer. 2009;45(2):228-47.

21. Kanda Y. Investigation of the freely available easy-to-use software 'EZR' for medical statistics. Bone Marrow Transplant. 2013;48(3):452-8.

22. Arcila ME, et al. EGFR exon 20 insertion mutations in lung adenocarcinomas: prevalence, molecular heterogeneity, and clinicopathologic characteristics. Mol Cancer Ther. 2013;12(2):220-9.

23. Choudhury NJ, et al. Response to Standard Therapies and Comprehensive Genomic Analysis for Patients with Lung Adenocarcinoma with EGFR Exon 20 Insertions. Clin Cancer Res. 2021;27(10):2920-7. 
24. Remon J, et al. EGFR exon 20 insertions in advanced non-small cell lung cancer: A new history begins. Cancer Treat Rev. 2020;90:102105.

25. Cardona AF, et al. EGFR exon 20 insertion in lung adenocarcinomas among Hispanics (geno1.2-CLICaP). Lung Cancer. 2018;125:265-72.

26. Wang Y, et al. Real-world treatment outcome of advanced Chinese NSCLC EGFR exon 20 insertion patients. J Clin Oncol. 2019;37(15_suppl):9043-3.

27. Qin Y, et al. Variability of EGFR exon 20 insertions in 24468 Chinese lung cancer patients and their divergent responses to EGFR inhibitors. Mol Oncol. 2020;14(8):1695-704.

28. Yasuda $\mathrm{H}$, et al. Structural, biochemical, and clinical characterization of epidermal growth factor receptor (EGFR) exon 20 insertion mutations in lung cancer. Sci Transl Med. 2013;5(216):216ra177.

29. Socinski MA, et al. Atezolizumab for First-Line Treatment of Metastatic Nonsquamous NSCLC. N Engl J Med. 2018;378(24):2288301.

30. Lee CK, et al. Checkpoint Inhibitors in Metastatic EGFR-Mutated Non-Small Cell Lung Cancer-A Meta-Analysis. J Thorac Oncol. 2017;12(2):403-7.

31. Gainor JF, et al. EGFR Mutations and ALK Rearrangements Are Associated with Low Response Rates to PD-1 Pathway Blockade in Non-Small Cell Lung Cancer: A Retrospective Analysis. Clin Cancer Res. 2016;22(18):4585-93.

32. Yamada T, et al. Retrospective efficacy analysis of immune checkpoint inhibitors in patients with EGFR-mutated non-small cell lung cancer. Cancer Med. 2019;8(4):1521-9.

33. Socinski MA, et al. LBA60 ZENITH20, a multinational, multi-cohort phase II study of poziotinib in NSCLC patients with EGFR or HER2 exon 20 insertion mutations. Ann Oncol. 2020;31:S1188.

34. Heymach J, et al. OA02.06 A Phase II Trial of Poziotinib in EGFR and HER2 exon 20 Mutant Non-Small Cell Lung Cancer (NSCLC). Journal of Thoracic Oncology. 2018;13(10):S323-4.

35. Jänne PA, et al., 1412TiP Mobocertinib (TAK-788) as first-line treatment vs platinum-based chemotherapy (CT) for NSCLC with EGFR exon 20 insertions (exon20ins). Annals of Oncology, 2020. 31: p. S892-S893.

\section{Supplementary Tables}

Supplemental Table 1. Clinical outcomes according to the variants of exon20 insertions.

\begin{tabular}{|llllllllllll|}
\hline No & sex & age & smoking & PS & stage & $\begin{array}{l}\text { Variants of Exon } \\
\mathbf{2 0} \text { insertion }\end{array}$ & $\begin{array}{l}\text { EGFR- } \\
\text { TKIs }\end{array}$ & $\begin{array}{l}\text { Chemotherapy } \\
\text { Best }\end{array}$ & $\begin{array}{l}\text { PFS } \\
\text { Response }\end{array}$ & $\begin{array}{l}\text { OS } \\
\text { (month) }\end{array}$ \\
\hline 1 & F & 52 & Never & 1 & IVA & A767_S768insTLA & N/A & CBDCA+PEM & SD & 17.3 & 18.3 \\
\hline 2 & F & 58 & Never & 1 & IVB & A767_V769dupASV & N/A & CDDP+PEM & SD & 14.2 & 29.3 \\
\hline 3 & F & 51 & Never & 0 & IVA & D770_771insASV & N/A & CDDP+PEM & SD & 12.5 & 39.7 \\
\hline 4 & F & 54 & Never & 1 & IVA & A767_V769dupASV & N/A & CDDP + PEM & PD & 2.5 & 14.1 \\
\hline \multicolumn{7}{|l}{ CDDP, cisplatin; CBDCA, carboplatin; PEM, pemetrexed; SD, stable disease; PD, progressive disease; N/A, not applicable. } \\
\hline
\end{tabular}

\section{Figures}


A.

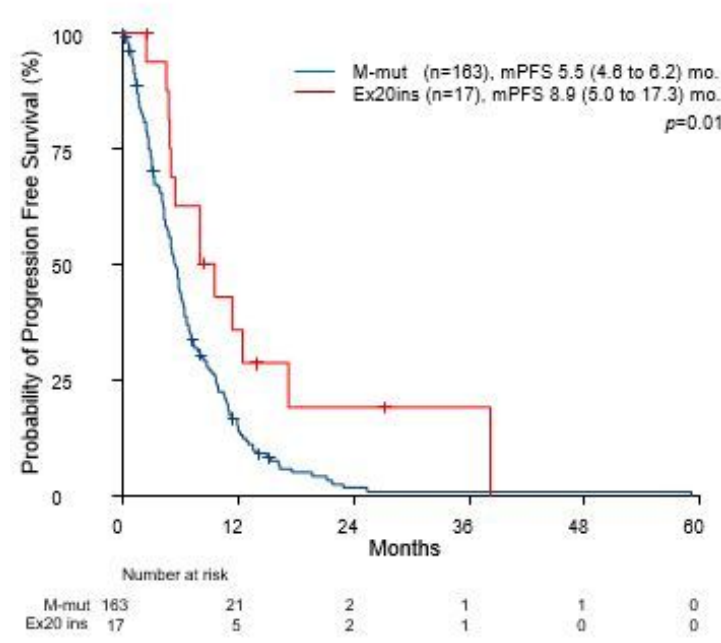

C.

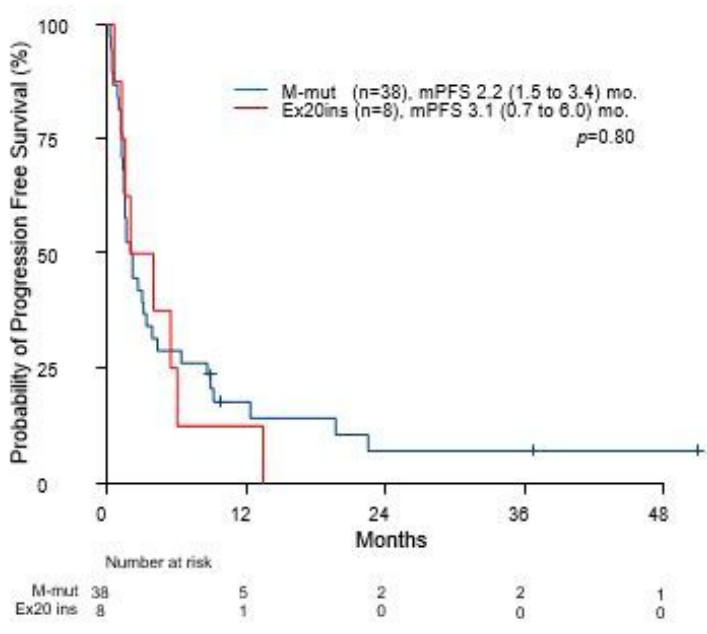

B.

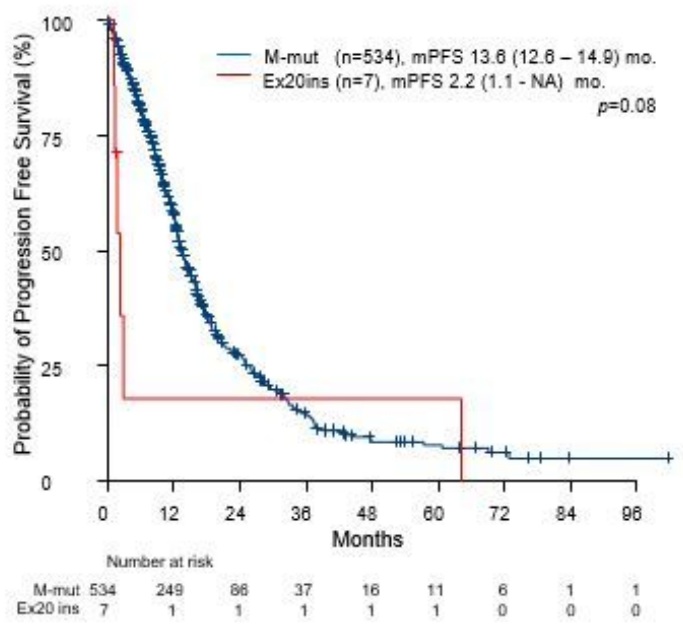

\section{Figure 1}

Median progression free survival after (A) platinum doublet chemotherapy, (B) EGFR-TKIs, and (C) anti-PD-1 antibody treatment in patients with EGFR exon 20 insertions and major mutations (L858R and exon 19 deletions). 


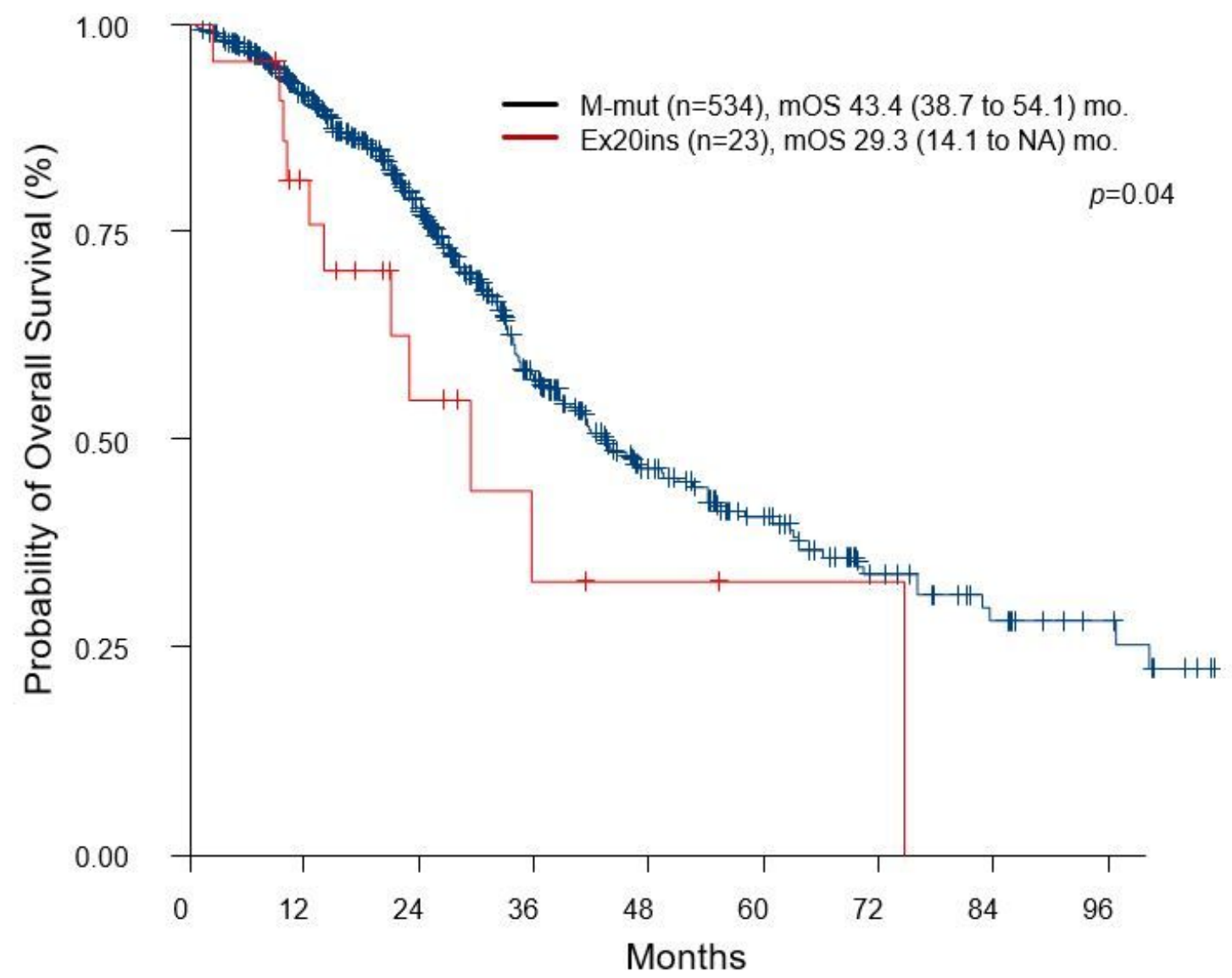

\section{Number at risk}

$\begin{array}{rccccccccc}\text { M-mut } & 534 & 418 & 280 & 155 & 89 & 56 & 30 & 18 & 11 \\ \text { Ex20ins } & 23 & 15 & 7 & 3 & 2 & 1 & 1 & 0 & 0\end{array}$

\section{Figure 2}

Overall survival in patients with EGFR exon 20 insertions and major mutations (L858R and exon 19 deletions). 